\title{
Charge response and energy calibration of ProtoDUNE-SP
}

Graham Chambers-Wall, William Jewell College, for the DUNE Collaboration Supervisors: Wanwei Wu and Tingjun Yang, Neutrino Division, Fermilab

FERMILAB-POSTER-20-078-ND

\section{Introduction}

ProtoDUNE-SP is a test bed liquid argon time projection chamber (LArTPC) for the far detector of the Deep Underground Neutrino Experiment (DUNE). This LArTPC was calibrated using cosmic-ray cathode-crossing muons, electric field maps, and purity-monitor data to correct for nonuniformities in the detector response and cosmic-ray stopping muons to perform the absolute energy scale calibration for further physics analysis.
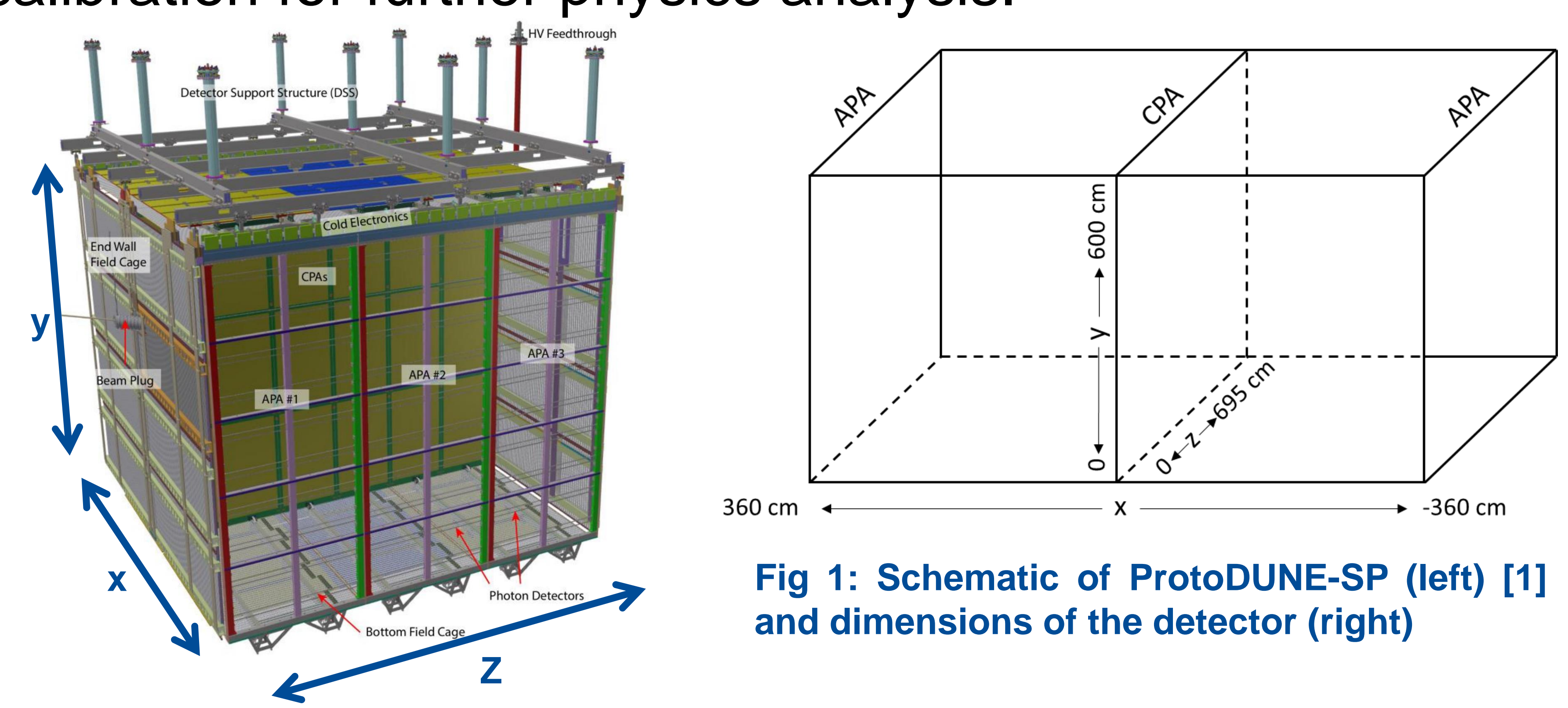

Fig 1: Schematic of ProtoDUNE-SP (left) [1] nd dimensions of the detector (right)

\section{Space Charge Effects}

Fig 2: Charge deposition per unit length $(d Q / d x)$ vs $x$ (SCE) corrections applied in plane 2 corge effec passing through the detector cause the accumulation of positive ions, leading to field in the LArTPC. SCE are corrected for using

\section{Electron Lifetime Correction}
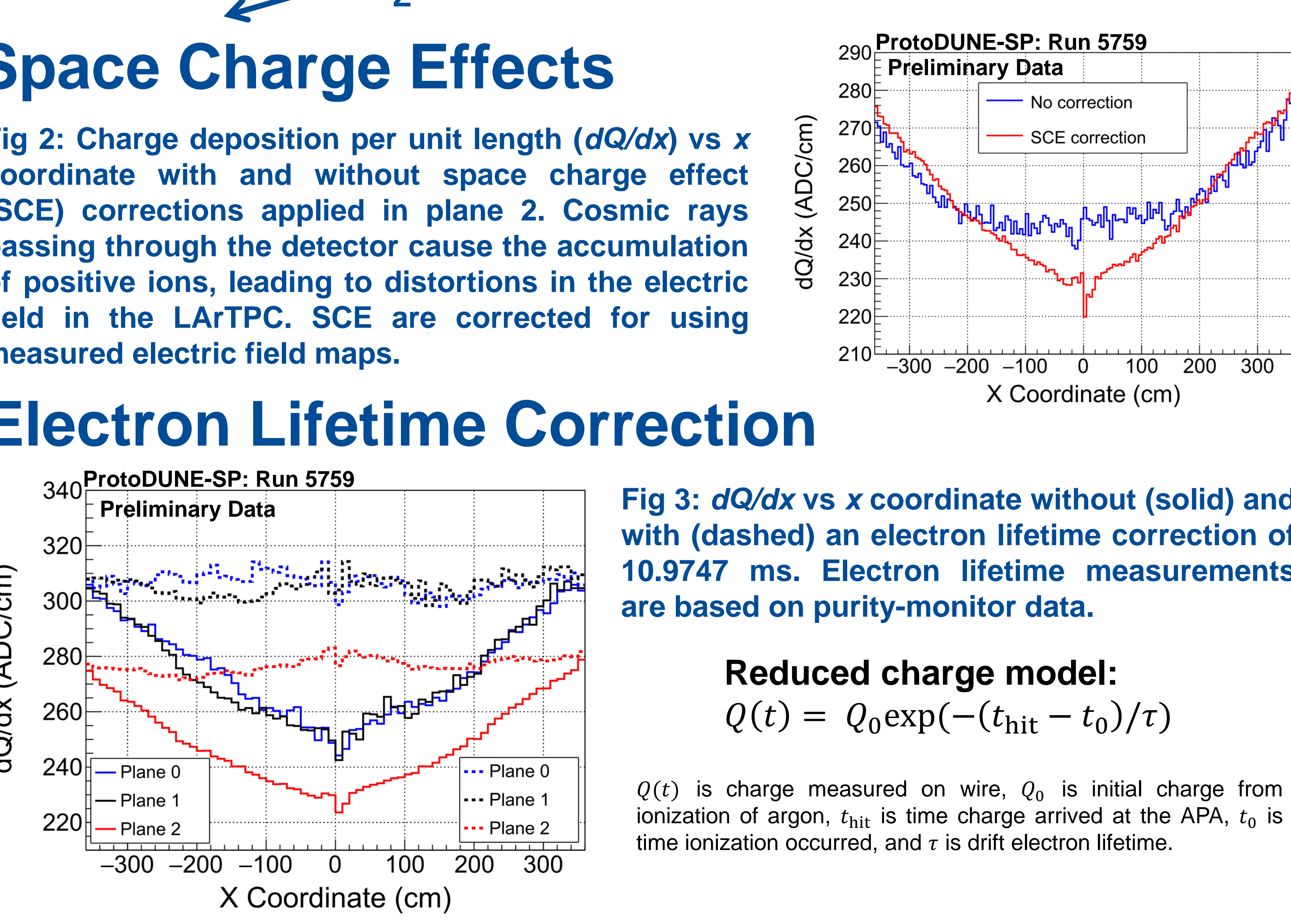

Fig 3: $d Q / d x$ vs $x$ coordinate without (solid) and with (dashed) an electron lifetime correction of $10.9747 \mathrm{~ms}$. Electron lifetime

Reduced charge model: $Q(t)=Q_{0} \exp \left(-\left(t_{\mathrm{hit}}-t_{0}\right) / \tau\right)$

$Q(t)$ is charge measured on wire, $Q_{0}$ is initial charge from
ionination of argon, thint is time charge arrivivd at the APA, $t_{0}$ is
time ionization occurred and $\tau$ is drift lectron lifetime. X Coordinate $(\mathrm{cm})$

\section{YZ, $\mathrm{X}$, and Normalization Corrections}

Divide two volumes into $5 \times 5 \mathrm{~cm}^{2}$ bins for $y z$ plane and $5 \mathrm{~cm}$ bins for $x$ coordinate Correction factors calculated using global $d Q / d x$ (median value across $x$ coordinate

Normalization using median $d Q / d x$ at anode and global $d Q / d x$

$$
\begin{array}{lll}
\text { YZ correction factor: } & \mathbf{X} \text { correction factor: } & \text { Normalization factor: } \\
C(y, z)=\frac{(d Q / d x)_{\mathrm{YZ}}^{\text {global }}}{(d Q / d x)_{\mathrm{YZ}}^{\text {lolal }}} & C(x)=\frac{(d Q / d x)_{\mathrm{X}}^{\text {lobal }}}{(d Q / d x)_{\mathrm{x}}^{\text {local }}} & N_{Q}=\frac{(d Q / d x)^{\text {node }}}{(d Q / d x)^{\text {global }}}
\end{array}
$$

$(d Q / d x)_{\text {calibrated }}=N_{Q} C(y, z) C(x)(d Q / d x)_{\text {reconstructed }}$
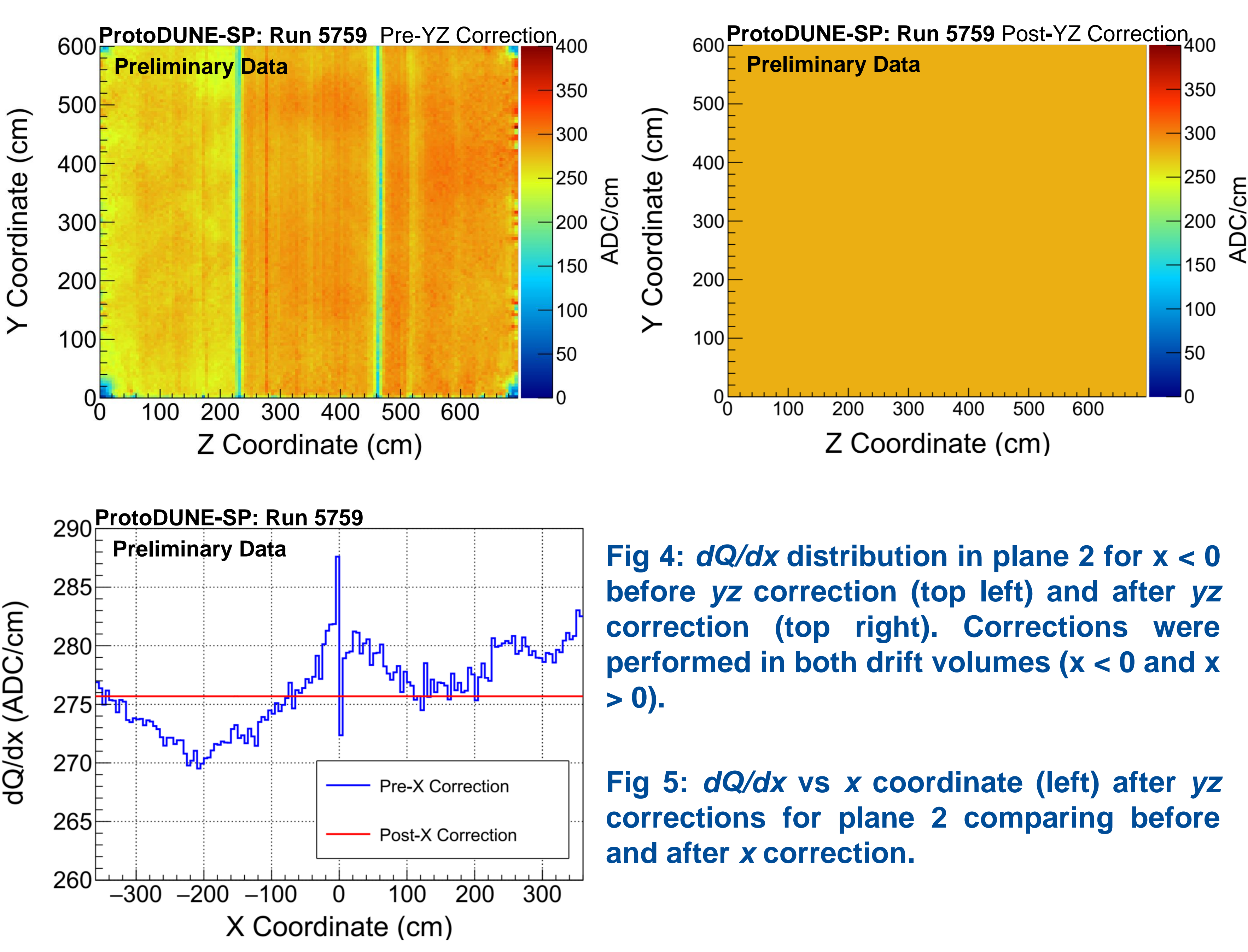

Fig 4: $d Q / d x$ distribution in plane 2 for $x<0$ before $y z$ correction (top left) and after $y z$ performed in both drift volumes $(x<0$ and $x$ $>0$ ).

Fig 5: $d Q / d x$ vs $x$ coordinate (left) after $y z$ corrections for plane 2 comparing before and after $x$ correction.

Charge Distributions
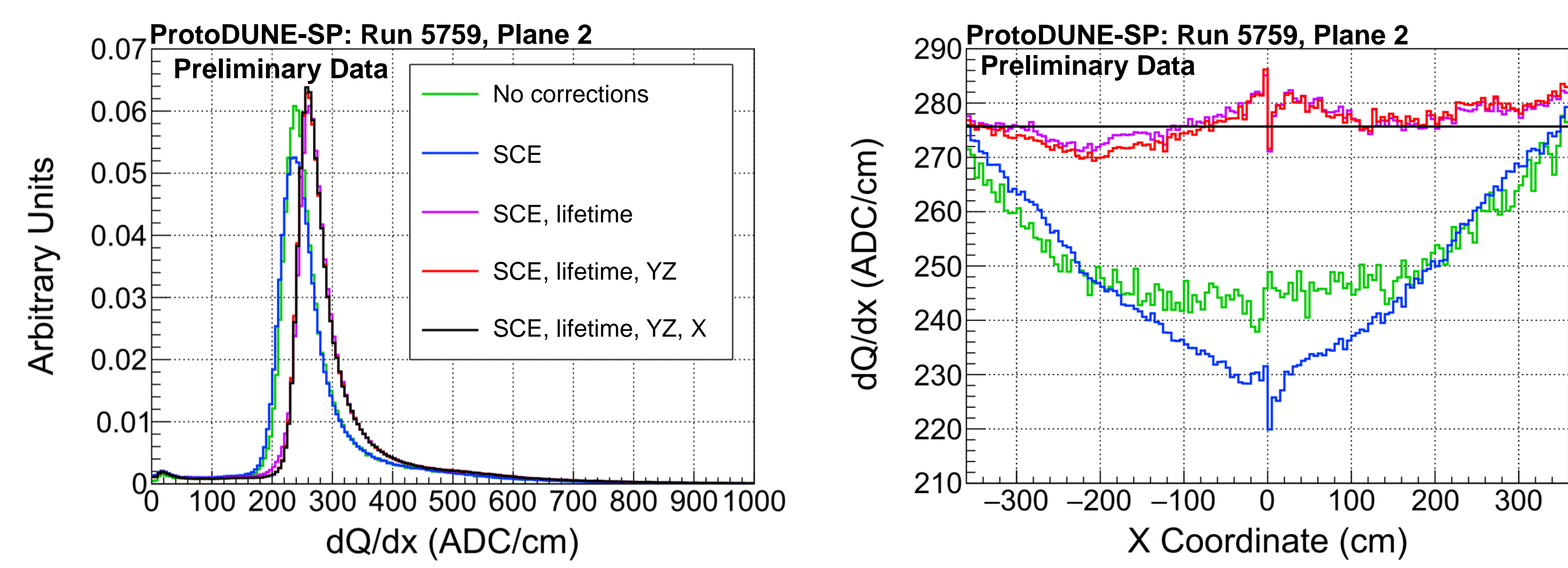

Fig 6: $d Q / d x$ vs $X$ (right) and $d Q / d x$ distribution (left) of cathode-crossing muons comparing no corrections, SCE correction,
corrections, and SCE/lifetime/YZ/X corrections.

\section{Absolute Energy Calibration}

Modified Box Model:

$\left(\frac{d E}{d x}\right)_{\text {calibrated }}=\left(\exp \left[\frac{\left(\frac{d Q}{d x}\right)_{\text {calibrated }}}{C_{\text {cal }}} \frac{\beta^{\prime} W_{\text {ion }}}{\rho \mathcal{E}}\right]-\alpha\right)\left(\frac{\rho \mathcal{E}}{\beta^{\prime}}\right)$ $C_{\text {cal }}=$ Constant used to convert ADC values to number of electrons,
$W_{\text {ion }}=23.6 \times 10^{-6}$ MeV Velectron (work function of argon),
$\mathcal{E} \quad=$ ProtoDUNE-SP $E$ field based on the space charge maps, $=1.38 \mathrm{~g} / \mathrm{cm}^{3}$ (liquid argon density at a pressure of $124.106 \mathrm{kPa}$ ) $\beta^{\prime} \quad=0.212(\mathrm{kV} / \mathrm{cm})\left(\mathrm{g} / \mathrm{cm}^{2}\right) / \mathrm{MeV}$, and
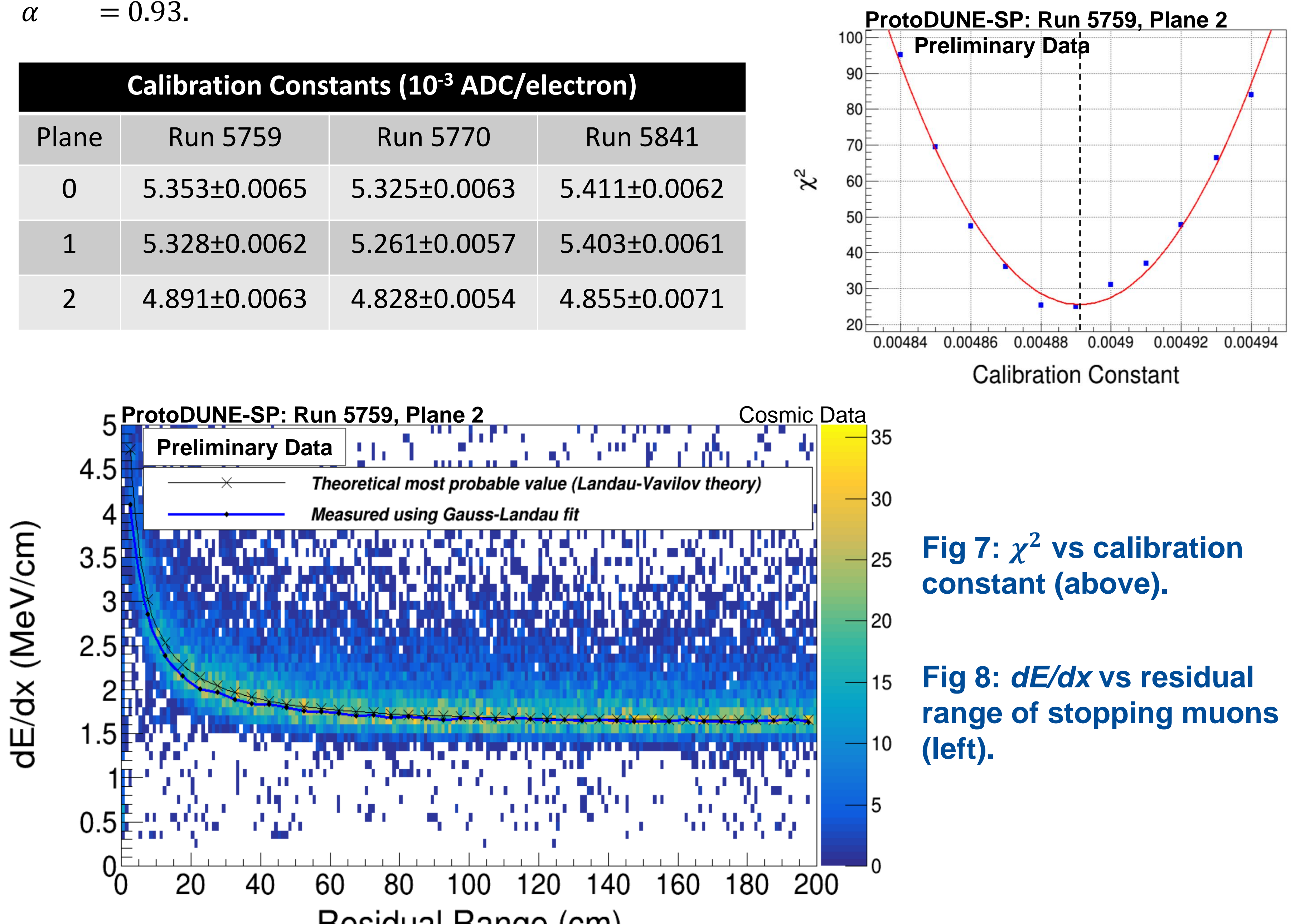

\section{Conclusions}

SCE lifetime corrections, YZ corrections, $X$ corrections, and normalization factors were applied to runs 5759,5770 , and 5841. Calibration constants were determined to convert $d Q / d x$ to $d E / d x$ for the absolute energy scale. These calibration factors were upload to a database for use in further physics analysis.

\section{References}

NNE Technical Design Report" arxiv: $1706.07081,2020$

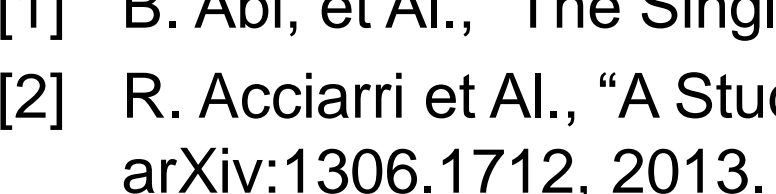

\section{Acknowledgements}

This work was supported in part by the U.S. Department of Energy, Office of Science, Office of Workforce

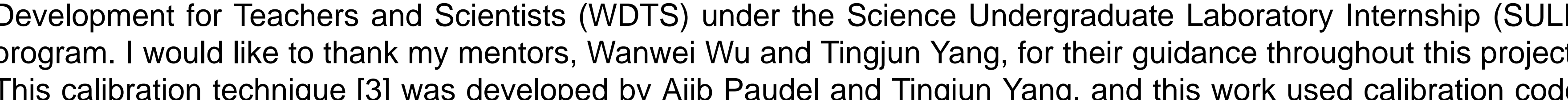
written by Ajib [4]. 